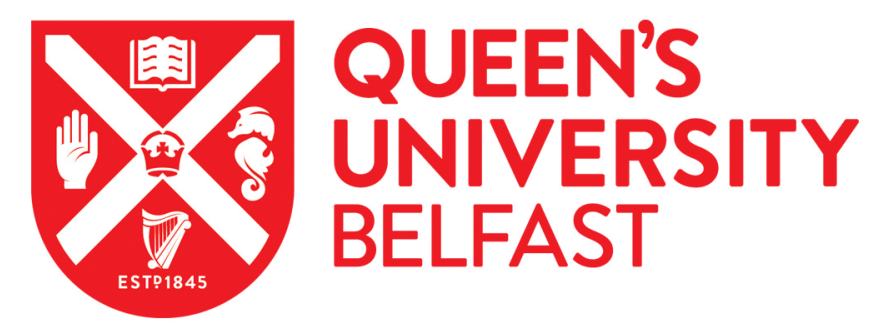

\title{
Epitaxial strain stabilization of a ferroelectric phase in PbZrO3 thin films
}

Chaudhuri, A. R., Arredondo, M., Hähnel, A., Morelli, A., Becker, M., Alexe, M., \& Vrejoiu, I. (2011). Epitaxial strain stabilization of a ferroelectric phase in PbZrO3 thin films. Physical Review B (Condensed Matter), 84(5), [054112]. https://doi.org/10.1103/PhysRevB.84.054112

Published in:

Physical Review B (Condensed Matter)

Document Version:

Publisher's PDF, also known as Version of record

Queen's University Belfast - Research Portal:

Link to publication record in Queen's University Belfast Research Portal

Publisher rights

Copyright 2011 American Physical Society.

This work is made available online in accordance with the publisher's policies. Please refer to any applicable terms of use of the publisher.

\section{General rights}

Copyright for the publications made accessible via the Queen's University Belfast Research Portal is retained by the author(s) and / or other copyright owners and it is a condition of accessing these publications that users recognise and abide by the legal requirements associated with these rights.

Take down policy

The Research Portal is Queen's institutional repository that provides access to Queen's research output. Every effort has been made to ensure that content in the Research Portal does not infringe any person's rights, or applicable UK laws. If you discover content in the Research Portal that you believe breaches copyright or violates any law, please contact openaccess@qub.ac.uk. 


\title{
@s \\ Epitaxial strain stabilization of a ferroelectric phase in $\mathrm{PbZrO}_{3}$ thin films
}

\author{
Ayan Roy Chaudhuri, Miryam Arredondo, Angelika Hähnel, Alessio Morelli, Michael Becker, Marin Alexe, and \\ Ionela Vrejoiu \\ Max Planck Institute of Microstructure Physics, Weinberg 2, D-06120 Halle (Saale), Germany \\ (Received 22 April 2011; revised manuscript received 23 June 2011; published 12 August 2011)
}

\begin{abstract}
$\mathrm{PbZrO}_{3} / \mathrm{SrRuO}_{3} / \mathrm{SrTiO}_{3}$ (100) epitaxial heterostructures with different thickness of the $\mathrm{PbZrO}_{3}(\mathrm{PZO})$ layer $\left(\mathrm{d}_{\mathrm{PZO}} \sim 5-160 \mathrm{~nm}\right)$ were fabricated by pulsed laser deposition. The ultrathin PZO films $\left(\mathrm{d}_{\mathrm{PZO}} \leqslant 10 \mathrm{~nm}\right)$ were found to possess a rhombohedral structure. On increasing the PZO film thickness, a bulk like orthorhombic phase started forming in the film with $\mathrm{d}_{\mathrm{PZO}} \sim 22 \mathrm{~nm}$ and became abundant in the thicker films. Nanobeam electron diffraction and room-temperature micro-Raman measurements revealed that the stabilization of the rhombohedral phase of PZO could be attributed to the epitaxial strain accommodated by the heterostructures. Room-temperature polarization vs electric field measurements performed on different samples showed characteristic double hysteresis loops of antiferroelectric materials accompanied by a small remnant polarization for the thick PZO films $\left(\mathrm{d}_{\mathrm{PZO}} \geqslant 50 \mathrm{~nm}\right)$. The remnant polarization increased by reducing the PZO layer thickness, and a ferroelectric like hysteresis loop was observed for the sample with $\mathrm{d}_{\mathrm{PZO}} \sim 22 \mathrm{~nm}$. Local ferroelectric properties measured by piezoresponse force microscopy also exhibited a similar thickness-dependent antiferroelectric-ferroelectric transition. Room-temperature electrical properties observed in the PZO thin films in correlation to their structural characteristics suggested that a ferroelectric rhombohedral phase could be stabilized in thin epitaxial PZO films experiencing large interfacial compressive stress.
\end{abstract}

DOI: 10.1103/PhysRevB.84.054112

PACS number(s): 77.84.-s, 77.80.B-, 77.55.-g

\section{INTRODUCTION}

Antiferroelectric materials (AFEs) in addition to ferroelectric materials (FEs) have attracted considerable research interest in the recent past. Thin films of AFEs have been extensively investigated for their potential applications in sensors, actuators, charge storage devices, and electro-optic devices. ${ }^{1-6}$ In recent years the giant electrocaloric effect observed in some AFEs projected them as potential candidates for application in electrical refrigeration devices. ${ }^{7,8}$ Besides their technological importance, AFEs are also interesting for fundamental studies. Below the Curie temperature $\left(\mathrm{T}_{\mathrm{C}}\right)$ AFEs contain oppositely oriented dipoles in the unit cell and, therefore, do not show any spontaneous polarization. Under a sufficiently large applied electric field, these materials undergo an electric field-driven transition to a FE phase that switches back to the AFE phase on removal of the electric field. Therefore, AFEs are commonly characterized by a double polarization hysteresis (P-E) loop combined with a zero remnant polarization $\left(\mathrm{P}_{\mathrm{r}}\right)$ under a sufficiently large applied electric field.

$\mathrm{PbZrO}_{3}(\mathrm{PZO})$ is the prototype of AFE materials with a $\mathrm{T}_{\mathrm{C}} \sim 230{ }^{\circ} \mathrm{C} .{ }^{9-12}$ At room temperature (RT), $\mathrm{PZO}$ possesses an orthorhombic structure $(a \sim 5.88 \AA, b \sim 11.78 \AA$, and $c \sim 8.22 \AA$ ) with the AFE axis lying in the $a b$-plane of the unit cell. The orthorhombic unit cell of PZO is commonly represented as a pseudocubic unit cell with $a_{p c} \sim 4.16 \AA$. $^{13}$ On application of a high electric field, PZO undergoes a structural phase transition from AFE orthorhombic $\left(\mathrm{AFE}_{\mathrm{O}}\right)$ to $\mathrm{FE}$ rhombohedral $\left(\mathrm{FE}_{\mathrm{R}}\right)$ and thereby exhibits the characteristic double P-E hysteresis loop. This electric field-induced transition in PZO is possible due to a small difference in the free energies of the $\mathrm{AFE}_{\mathrm{O}}$ and $\mathrm{FE}_{\mathrm{R}}$ phases. ${ }^{14-16}$ Jona et al. reported that, in addition to antiferroelectricity, PZO might also possess ferroelectricity along the $[001]_{\mathrm{O}}$ axis of the unit cell with a large $\mathrm{P}_{\mathrm{r}}$ of $25 \mu \mathrm{C} / \mathrm{cm}^{2}$ due to the unbalanced antiparallel displacement of the oxygen ions. ${ }^{17}$ Due to the possible coexistence of both antiferroelectricity and ferroelectricity, the term ferrielectric was also proposed to describe PZO, in slight analogy with ferrimagnetism. ${ }^{9}$ Later, Dai et al. demonstrated very weak RT ferroelectricity in PZO ceramic with a $\mathrm{P}_{\mathrm{r}} \sim 0.1 \mu \mathrm{C} / \mathrm{cm}^{2}{ }^{18}$ Unfortunately, no further report on RT ferroelectricity in ceramic or single-crystal PZO is available to date. Pintilie et al. reported low-temperature ferroelectricity with large $\mathrm{P}_{\mathrm{r}}$ value $\left(27 \mu \mathrm{C} / \mathrm{cm}^{2}\right)$ in epitaxial PZO $(001)_{\mathrm{O}}$ thin films. ${ }^{19}$ Manifestation of ferroelectricity in high-purity ceramics and single crystals of PZO was also observed in a narrow temperature range near the $\mathrm{T}_{\mathrm{C}}$, and in these cases the $\mathrm{AFE}_{\mathrm{O}}$ to paraelectric-cubic $\left(\mathrm{PE}_{\mathrm{C}}\right)$ phase transition was found to be associated with an intermediate $\mathrm{FE}_{\mathrm{R}}$ phase. ${ }^{20-23}$ Room-temperature ferroelectricity in PZO was demonstrated by Ayyub et $a l .^{24}$ for thin films below a critical thickness of $\sim 500 \mathrm{~nm}$ fabricated on $p$-type $\mathrm{Si}$ substrates and was explained in terms of the effective built in electric field at the PZO and Si interface. Various research groups reported that such size-dependent physical properties in different materials are associated with a structural phase transition, which can be achieved by stressing their epitaxial thin films through effective misfit. ${ }^{25-27}$ Boldyreva et al. ${ }^{28}$ reported an AFE-to-FE, orthorhombic-to- rhombohedral phase transition of the PZO layer at RT in case of PZO and $\mathrm{Pb}\left(\mathrm{Zr}_{0.8} \mathrm{Ti}_{0.2}\right) \mathrm{O}_{3}$ multilayered epitaxial heterostructures below a critical individual layer thickness of $\sim 10 \mathrm{~nm}$. Although the investigation successfully demonstrated that the FE property of the heterostructures is related to a rhombohedral phase, the origin of the phase stabilization could not be attributed unambiguously to the interfacial strain. Considering all the previous reports, it appears that stabilization of the $\mathrm{FE}_{\mathrm{R}}$ phase of PZO at RT without an electric field remains hitherto unachieved in single-layer $\mathrm{PZO}$ epitaxial thin films. 
To investigate the manifestation of thickness-dependent RT ferroelectricity in single-layer PZO thin films under epitaxial strain, we fabricated epitaxial PZO thin films of different thickness. Their structure, mechanical stress state, and electrical properties were investigated. The appearance of ferroelectricity in thin PZO films has been discussed in close correlation to their microstructure and the effect of mechanical stress field present at the film-substrate interface.

\section{EXPERIMENTAL}

In the present study, vicinal single crystalline $\mathrm{SrTiO}_{3}(100)$ (STO, $a=3.905 \AA$ ) substrates (CrysTec GmbH, Berlin) with a miscut angle of about $0.1^{\circ}$ were used for growing the thin films. In order to achieve $\mathrm{TiO}_{2}$-terminated surfaces, the STO substrates were etched in a buffered HF solution and annealed in air at a temperature of $950{ }^{\circ} \mathrm{C} .^{29}$ This treatment resulted in one-unit cell-stepped terraces with straight ledges. Ceramic targets of $\mathrm{SrRuO}_{3}$ (SRO) and PZO were used for preparing the thin films. A series of PZO films of various thickness $\left(\mathrm{d}_{\mathrm{PZO}} \sim 5-160 \mathrm{~nm}\right)$ were fabricated on a $\sim 20$-nm-thick SRO layer by pulsed laser deposition (PLD) using a $\mathrm{KrF}$ excimer laser (Lambda Physik, LPX, $\lambda=248 \mathrm{~nm}$ ). The base pressure of the chamber was brought down to $\sim 1 \times 10^{-6}$ mbar prior to each deposition. The films were deposited under oxygen partial pressure of $0.14 \mathrm{mbar}$, with laser fluence of $1-1.5 \mathrm{~J} / \mathrm{cm}^{2}$ at a laser repetition rate, $v \sim 5 \mathrm{~Hz}$, keeping the target to substrate distance $\left(\mathrm{d}_{\mathrm{T}-\mathrm{S}}\right) \sim 5.5 \mathrm{~cm}$. The substrate temperature was maintained at $700{ }^{\circ} \mathrm{C}$ for depositing the SRO layer, whereas for growing the PZO layer the temperature was set at $575^{\circ} \mathrm{C}$. The films were brought back to RT immediately after deposition with a cooling rate of $10^{\circ} \mathrm{C} \mathrm{min}^{-1}$. SRO top electrodes of area $\sim 0.0036 \mathrm{~mm}^{2}$ were deposited by PLD at RT using a shadow mask. Pt was sputtered on top of the SRO electrodes in order to facilitate the contact to the capacitors for electrical characterizations.

The surface morphologies of the substrate and the grown films were studied by atomic force microscopy (AFM) in tapping mode using a D5000 microscope (Digital Instruments). For crystallographic characterizations of the thin films, $\mathrm{X}$-ray diffraction (XRD) measurements were performed using a four-circle diffractometer $\left(\mathrm{Cu} \mathrm{K} \mathrm{K}_{\alpha}, \lambda=0.15418 \mathrm{~nm}\right.$ ) (X'Pert MRD, Philips). Transmission electron microscopy (TEM) investigations were performed to find out the microstructural details of the heterostructures. TEM samples were prepared by standard mechanical and ion beam thinning procedures. ${ }^{30}$ High-resolution TEM (HRTEM) investigations were performed in a JEOL 4010 microscope (JEOL GmbH) operated at $400 \mathrm{kV}$. For the nanobeam electron diffraction (NBED) measurements, a probe $\mathrm{C}_{\mathrm{s}}$-corrected FEI-Titan 80300 electron microscope (FEI Company) was employed, which was operated at $300 \mathrm{kV}$ with a $20-\mu \mathrm{m}$ second condenser aperture. The aperture defines both the semi-convergence angle of $0.3 \mathrm{mrad}$ and the full width at half maximum (FWHM) of the illuminating electron beam of about $3 \mathrm{~nm}$ in the micro probe STEM mode. Series of diffraction patterns were acquired point-by-point with a $2 \mathrm{k} \times 2 \mathrm{k}$ charge coupled device (CCD) camera being integrated into the FEI-TITAN.
For analyzing the series of diffraction patterns, a commercial software package was used, which precisely determines the centers of the diffraction spots and the displacement of their positions in relation to reference patterns acquired in an unstrained area of the sample. ${ }^{31}$ Micro-Raman-spectroscopy was used to detect the mechanical stress states at the interface region of the PZO films. The Raman measurements were performed at RT using a LabRAM HR 800 spectrometer (Horiba Jobin Yvon $\mathrm{GmbH}$ ) equipped with a $\mathrm{HeCd}$ laser with the main emission line at $325 \mathrm{~nm}(\sim 3.815 \mathrm{eV})$. The energy of the excitation line was chosen to be larger than the band gap of PZO $(\sim 3.7 \mathrm{eV})^{32,33}$ to limit the penetration depth of the laser beam only to a few nanometers from the film surface, thereby enabling the stress analysis only of the PZO films. The spot diameter on the samples was $\sim 3 \mu \mathrm{m}$ and the laser power was $<1 \mathrm{~mW}$, resulting in a small energy density, such that effects due to sample heating could be safely ignored. For the Raman-based stress analysis, the mode located at $\sim 415 \mathrm{~cm}^{-1}$ in stress-free bulk PZO was selected, and the amount of stress was calculated from the shift of the peak with change in $\mathrm{d}_{\mathrm{PZO}} \cdot{ }^{34,35}$ RT polarization characteristics of the heterostructures were investigated by measuring their macroscopic dynamic P-E and static P-E hysteresis responses ${ }^{36}$ using an AixAcct TF analyzer 2000 (aixACCT Systems GmbH). In case of the dynamic P-E hysteresis measurements, the nonzero leakage current at small applied voltages adds a parasitic contribution to the integrated charge, which might yield a spurious value of the in-field $\mathrm{P}_{\mathrm{r}}{ }^{37}$ To isolate the true remnant component of the heterostructures from the nonremnant component, which remain mixed in the standard dynamic P-E measurement, remnant hysteresis measurements ${ }^{38}$ were performed using a precision multiferroic tester (Radiant Technologies, Inc.). In order to eliminate any experimental artifact, all the measurements were performed on several capacitors on the same samples. Local ferroelectric characteristics of the heterostructures were also investigated utilizing piezoresponse force microscopy (PFM). In this study both the in-field and remnant piezoelectric hysteresis loops ${ }^{39}$ were measured using a commercial scanning probe microscope (XE-100, Park Systems) and lock-in amplifier (SR850, Stanford Research System). DC leakage current in the samples was measured at RT using a Source Measure Unit (2635A, Keithley Instruments $\mathrm{GmbH}$ ).

\section{RESULTS AND DISCUSSIONS}

Figure 1 shows the XRD pattern in the $\theta-2 \theta$ geometry of four representative samples possessing different thickness of the PZO layer. All the samples exhibited epitaxial growth of the heterostructures without any impurity phase. Interestingly, the peak corresponding to PZO shifted towards higher $2 \theta$ angle with the reduction of $\mathrm{d}_{\mathrm{PZO}}$ below $50 \mathrm{~nm}$. The outof-plane lattice spacing of PZO extracted from the secondorder XRD peaks exhibited a thickness-dependent decrease from $\sim 4.16( \pm 0.1) \AA\left(\mathrm{d}_{\mathrm{PZO}} \sim 80 \mathrm{~nm}\right)$ to $\sim 4.11( \pm 0.1) \AA$ $\left(\mathrm{d}_{\mathrm{PZO}} \sim 8 \mathrm{~nm}\right)$. Similar observation was reported by Boldyreva et al. for PZT and PZO multilayered heterostructures and was attributed to the stabilization of a rhombohedral phase in $\mathrm{PZO} .{ }^{28}$ 


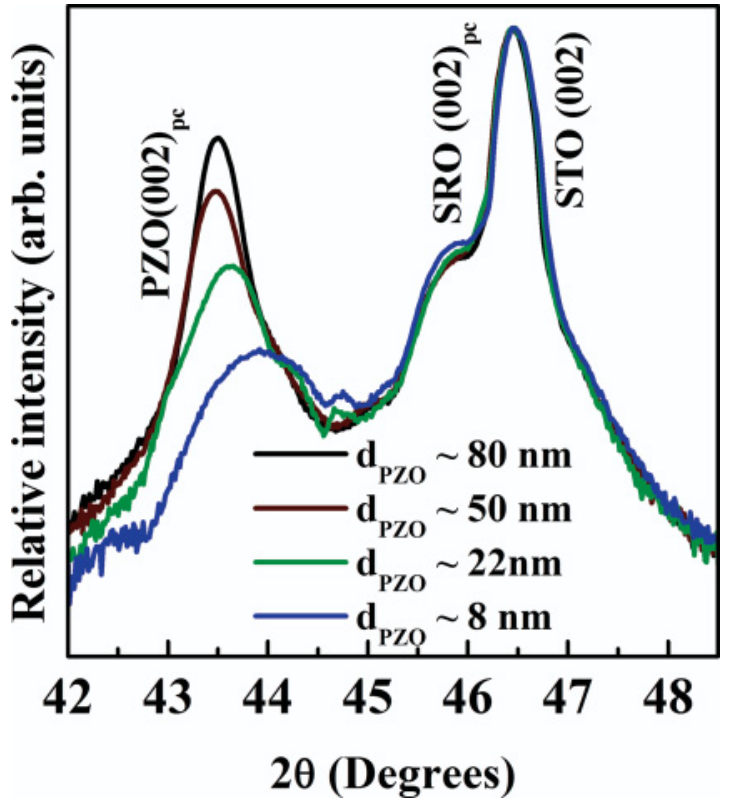

FIG. 1. (Color online) X-ray diffraction pattern of four representative PZO thin films exhibiting a gradual shift of the peak corresponding to PZO towards higher $2 \theta$ angle with decreasing the PZO layer thickness.

In case of epitaxial thin films, the lattice misfit strain tends to increase by reducing the film thickness. For thin films under compressive stress (as in the present case), such an increase in misfit strain is commonly associated with an increase in the out-of-plane lattice parameter, provided the phase and the orientation of the film remain unchanged. Therefore, our observation, which showed an opposite trend, primarily indicates a possible size-dependent change in the phase and/or orientation of the PZO thin films.

In order to explore the structure of the PZO thin films and their epitaxial relation to the substrate, microstructural investigations were carried out by detailed TEM and HRTEM studies. Figure 2 displays the bright-field HRTEM image of the sample with $\mathrm{d}_{\mathrm{PZO}} \sim 8 \mathrm{~nm}$. The TEM image clearly shows the epitaxial growth of the PZO and SRO layer on the substrate. Electron diffraction and fast Fourier transform (FFT) analyses of the HRTEM image established the epitaxial relationship (100) PZO || (100) SRO || (100) STO; [001] PZO || [001] SRO $\|$ [001] STO and yielded a rhombohedral structure of the PZO layer $\left(\mathrm{PZO}_{\mathrm{R}}\right)$ with $a \sim 4.11 \AA$ and $\beta \sim 89.85^{\circ}$. Additionally, edge-type dislocations were observed in this heterostructure. The dislocation cores were found to lie inside the SRO layer, close to the PZO and SRO interface, with a spacing of 5-10 nm and a Burgers vector $\mathbf{b}=\mathrm{a}[110]$ (see the marked Burgers circuit around one of the dislocations in the micrograph shown in Fig. 2). Considering the large theoretical lattice mismatch $(\sim-5.5 \%)$ between PZO and SRO, the presence of dislocations with a small spacing of 5-10 nm, indicating thus a large density of dislocations, is expected in these heterostructures. ${ }^{40}$ TEM investigations performed on different samples involving PZO layer thickness, ranging between 5 and $10 \mathrm{~nm}$, showed an identical rhombohedral structure for PZO.

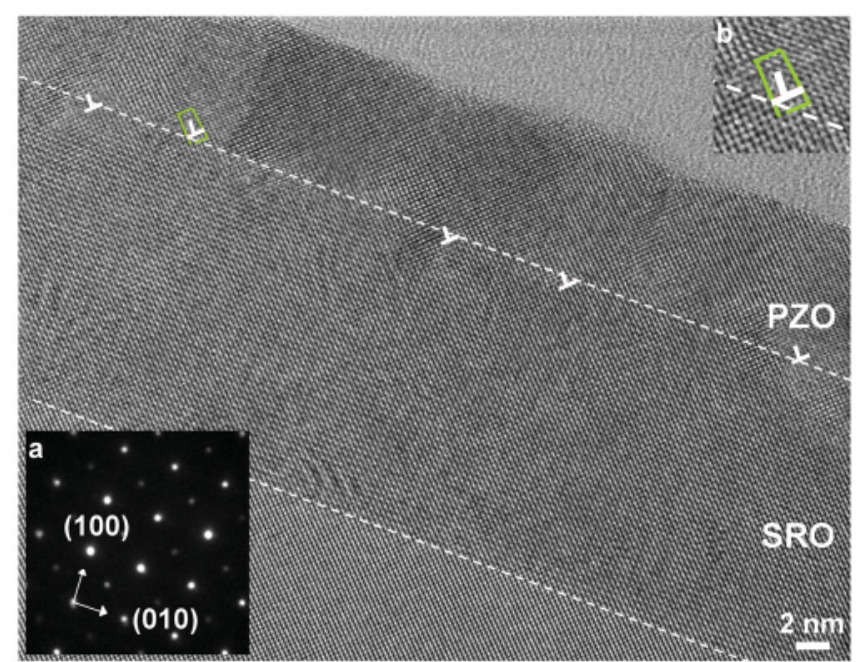

FIG. 2. (Color online) Cross-sectional HRTEM image of a heterostructure with $\mathrm{d}_{\mathrm{PZO}} \sim 8 \mathrm{~nm}$. The electron diffraction pattern corresponding to PZO [inset (a)] exhibits a rhombohedral symmetry. The positions of the misfit dislocations are marked in the image and a corresponding Burgers circuit is shown in the inset (b).

Figure 3 displays the HRTEM images of a sample with $\mathrm{d}_{\mathrm{PZO}} \sim 22 \mathrm{~nm}$. The PZO layer in this sample was found to possess an epitaxial relationship (100) $\mathrm{PZO}_{\mathrm{R}} \|$ (100) SRO \| (100) STO; [001] $\mathrm{PZO}_{\mathrm{R}} \|$ [001] SRO\| [001] STO. Edge dislocations with a spacing of 5-10 $\mathrm{nm}$ and $\mathbf{b}=\mathrm{a}[110]$ were also observed in this sample, similar to the sample with $\mathrm{d}_{\mathrm{PZO}} \sim 8 \mathrm{~nm}$. However, a closer look on different areas of the PZO layer indicated the formation of an orthorhombic phase of $\mathrm{PZO}\left(\mathrm{PZO}_{\mathrm{O}}\right)$ in addition to the $\mathrm{PZO}_{\mathrm{R}}(100)$ phase. In Fig. 3 the area marked by the white boxes and labeled as " $O$ " could be identified as $\mathrm{PZO}_{\mathrm{O}}$ (120) with $a \sim 5.51 \AA, b \sim 11.77 \AA$, and $c \sim 8.4 \AA$. On the other hand, the areas labeled as " $R$ " possessed a rhombohedral structure. Detailed TEM analyses of the sample revealed a rhombohedral phase up to a distance of $\sim 8 \mathrm{~nm}$ from the PZO and SRO interface and a bulklike orthorhombic

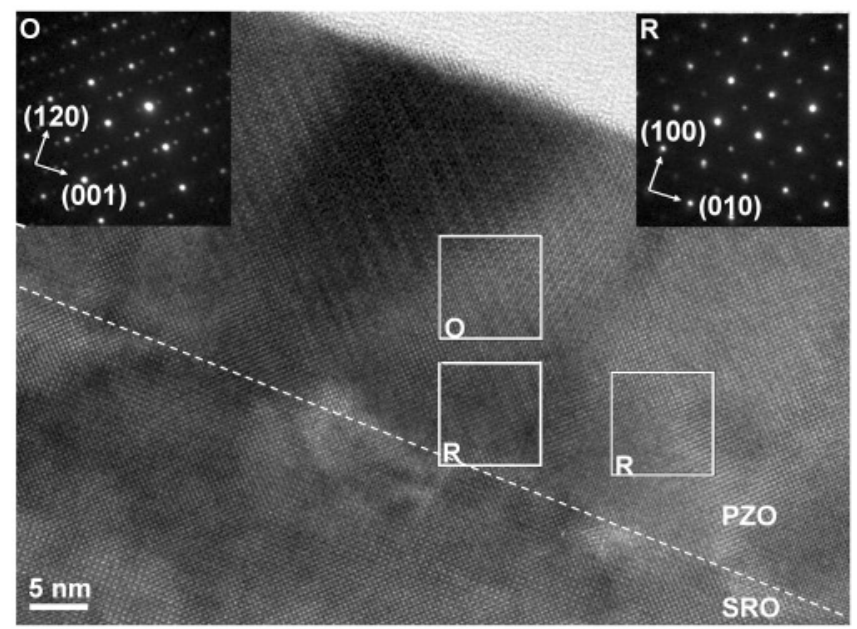

FIG. 3. Cross-sectional HRTEM image of the heterostructure with $\mathrm{d}_{\mathrm{PZO}} \sim 22 \mathrm{~nm}$. The electron diffraction patterns inset show the rhombohedral and the orthorhombic phases of PZO. 

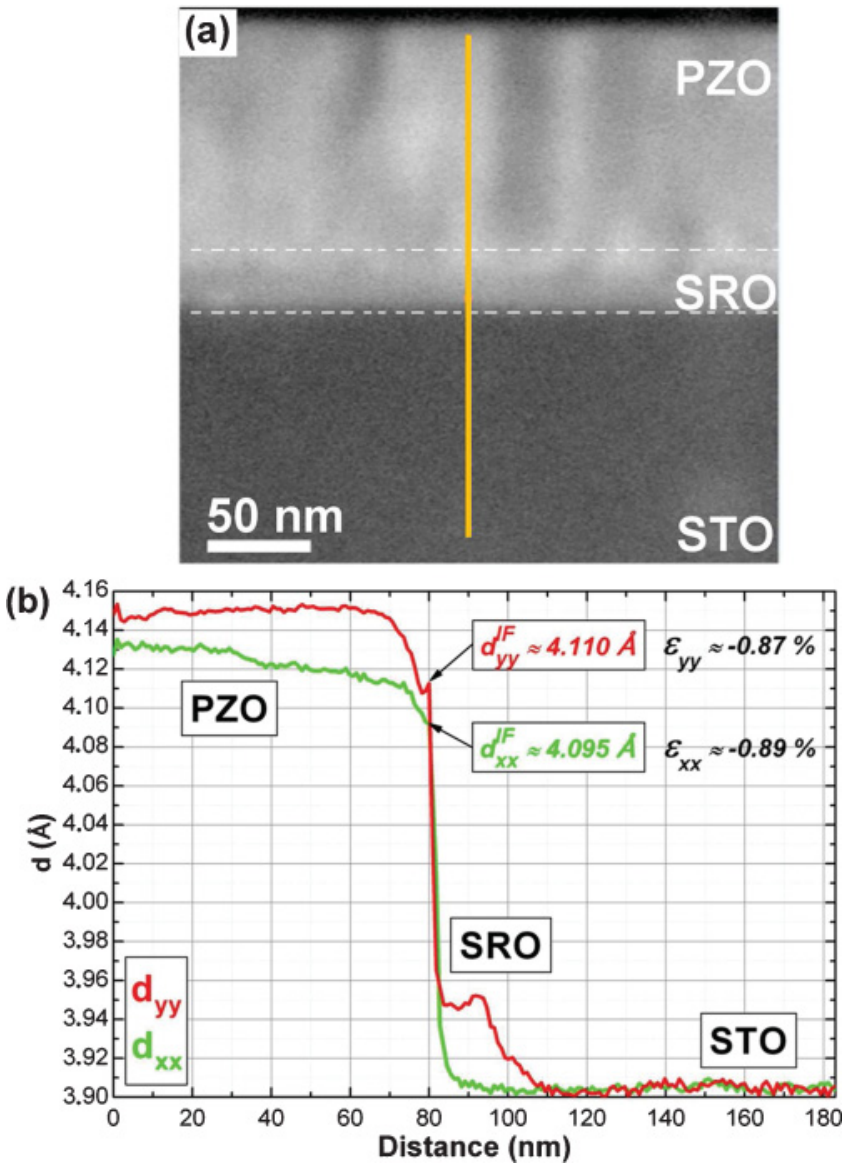

FIG. 4. (Color online) (a) STEM image of a representative PZO thin film $\left(\mathrm{d}_{\mathrm{PZO}} \sim 80 \mathrm{~nm}\right)$. The orange line indicates the location where the NBED pattern series was measured across the interfaces. (b) Lattice parameters corresponding to the different phases of PZO ( $p c$ $\mathrm{PZO}_{\mathrm{O}}$ and $\mathrm{PZO}_{\mathrm{R}}$ ) across the sample thickness; calculated from the NBED measurements.

phase thereafter. TEM investigations performed on different heterostructures with larger $\mathrm{d}_{\mathrm{PZO}}$ indicated the abundance of the $\mathrm{PZO}_{\mathrm{O}}$ phase, having the film substrate orientation relation $(120)_{\mathrm{o}} \mathrm{PZO}\|(100) \mathrm{SRO}\|(100) \mathrm{STO} ;[1-20]_{\mathrm{o}} \mathrm{PZO}_{\mathrm{O}} \|$ [001] SRO \| [001] STO.

Our TEM studies suggested a thickness-dependent rhombohedral to orthorhombic phase transition in the epitaxial PZO thin films under investigation. The manifestation of the $\mathrm{PZO}_{\mathrm{R}}$ and $\mathrm{PZO}_{\mathrm{O}}$ phases in the heterostructures over different thickness ranges could possibly be related to the epitaxial strain in the films. To gain insight into the strain state of the heterostructures NBED measurements were performed. During NBED, a selected nanometer-sized area of the specimen (being in zone axis orientation) was illuminated with a nearly parallel electron beam, and a series of diffraction patterns was acquired at different points along the lines defined beforehand. The strain components of interest were then calculated using a dedicated software package. ${ }^{31}$ From the strain profiles, which show the changes of the in-plane $\left(\mathrm{d}_{\mathrm{xx}}\right)$ and out-of-plane $\left(\mathrm{d}_{\mathrm{yy}}\right)$ lattice distances across the whole layer system, it was possible to estimate the corresponding absolute values of $d_{x x}$ and $d_{y y}$ by referring to the lattice parameters of the bulk cubic STO substrate $(a=3.90528 \AA)$. Figure 4(a) shows a STEM image of a representative 80-nm-thick $\mathrm{PZO}$ layer containing both the $\mathrm{PZO}_{\mathrm{R}}$ and $\mathrm{PZO}_{\mathrm{O}}$ phases. On panel Fig. 4(b), the corresponding $\mathrm{d}_{\mathrm{xx}}$ and $\mathrm{d}_{\mathrm{yy}}$ are plotted as a function of the distance on the measuring line. Near the surface of the sample, the lattice spacings of the PZO layer were found to be close to the pseudocubic lattice parameter of bulk PZO $\left(\mathrm{d}_{\mathrm{yy}} \sim 4.15 \AA\right.$ and $\mathrm{d}_{\mathrm{xx}} \sim 4.13 \AA$, in comparison to $a_{p c \text {-bulk }} \sim 4.16 \AA$ ). The subtle difference in the reported lattice parameters are mainly caused by slightly varying lattice parameters in the PZO volume and a measurement uncertainty of $\sim 0.1-0.2 \%$. From the surface to a depth of $\sim 60 \mathrm{~nm}$ the $\mathrm{d}_{\mathrm{yy}}$-profile shows a plateau, whereas the profile of $\mathrm{d}_{\mathrm{xx}}$ demonstrates two terraces separated by a step, which might correspond to orthorhombic defect structures. ${ }^{41}$ The regions of constant lattice distances indicate relaxation of strain in more than half of the PZO layer. However, from a depth of $\sim 60-70 \mathrm{~nm}$ up to the PZO and SRO interface, $\mathrm{d}_{\mathrm{yy}}$ and $\mathrm{d}_{\mathrm{xx}}$ continuously decrease to $4.11 \AA$ and $4.095 \AA$, respectively, which correspond to compressive out-of-plane and in-plane strain of $\sim-0.9 \%$ with respect to the surface of the PZO layer. Furthermore, it is important to note the distinct shoulder in the $\mathrm{d}_{\mathrm{yy}}$ profile adjacent to the interface between PZO and SRO. The above-mentioned lattice distances being associated to this shoulder in the $\mathrm{d}_{\mathrm{yy}}$ profile demonstrate the stabilization of the rhombohedral PZO phase $\left(a \sim 4.11 \AA\right.$ and $\left.\beta \sim 89.85^{\circ}\right)$ near the interface. The lattice parameters of the PZO layer calculated from the NBED and HRTEM investigations matched well with the values estimated from $\mathrm{x}$-ray reciprocal space analyses of the heterostructures (not shown here). These results clearly indicate the epitaxial strain-driven stabilization of the $\mathrm{PZO}_{\mathrm{R}}$ phase in the heterostructures under investigation. With lowering the thickness of the PZO layer, the strain field is expected to be extended uniformly over the entire layer thickness, resulting in the stabilization of only the rhombohedral phase.

It is worth mentioning here that we have not performed a detailed analysis of the stoichiometry of the PZO films. Therefore, the possible contribution of $\mathrm{Pb}$ and oxygen vacancies, which are often present in $\mathrm{Pb}$-based oxidic compounds, to the observed behavior of both the in-plane and out-of-plane lattice parameters of the PZO films cannot be completely ruled out. ${ }^{42}$

In order to further characterize the strain behavior in dependence on the layer thickness, RT micro-Raman spectroscopy was performed. Raman analyses of the films with $\mathrm{d}_{\mathrm{PZO}} \sim 12-$ $160 \mathrm{~nm}$ exhibited spectral features solely corresponding to the pure PZO Raman spectrum without any signal from the substrate and the buffer layer. The appearance of a Raman signal corresponding to the STO substrate and a marginal signal from the SRO layer for a sample with $\mathrm{d}_{\mathrm{PZO}} \sim 5 \mathrm{~nm}$ confirmed that the penetration depth of the $325 \mathrm{~nm}$ excitation line in PZO lies in the narrow range of 5-12 nm.

The film-thickness dependence of the stress values were determined from the Raman peak shifts following the linear peak shift vs mechanical stress curve for PZO reported by Furuta et $a .^{35}$ A shift of $2.9 \mathrm{~cm}^{-1}$ of the Raman peak at $415 \mathrm{~cm}^{-1}$ corresponded to a stress value of $1 \mathrm{GPa}$ in the present case. Figure 5 displays the Raman spectra of the heterostructures possessing different $d_{\mathrm{PZO}}$. The maxima position for $\mathrm{d}_{\mathrm{PZO}} \geqslant 80 \mathrm{~nm}$ matched with the peak observed in a stress-free bulk PZO ceramic (not shown here) indicating that the stress field inside the PZO layer has disappeared at 


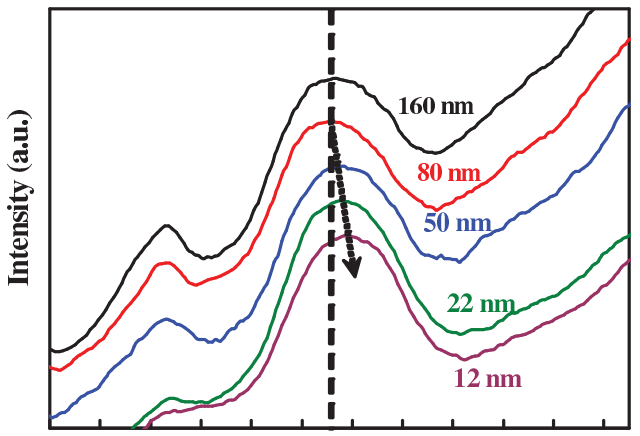

$\begin{array}{llllllllllll}300 & 320 & 340 & 360 & 380 & 400 & 420 & 440 & 460 & 480 & 500 & 520\end{array}$ Wavenumber $\left(\mathrm{cm}^{-1}\right)$

FIG. 5. (Color online) Raman spectra of different $\mathrm{PbZrO}_{3}$ film samples in the range of the $415 \mathrm{~cm}^{-1}$ Raman peak. The straight dotted line indicates the peak position corresponding to the 160- and 80 -nm-thick PZO films. The oblique arrow indicates the shift of the peak position by reducing the PZO layer thickness.

a distance $>80 \mathrm{~nm}$ from the PZO and SRO interface. The Raman peak shifted towards higher wave numbers due to the influence of the increasing compressive stress field in the PZO layer by reducing $d_{\text {PZO }}$ below $80 \mathrm{~nm}$. The (compressive) stress values, calculated from the Raman frequency shifts $\Delta \omega$, are graphically displayed in Fig. 6. Considering the linear relation between the mechanical stress and $d_{\mathrm{PzO}}$ observed in the present case, a stress value of $\sim 2.5 \mathrm{GPa}$ was estimated for the thin film with $\mathrm{d}_{\mathrm{PZO}} \sim 5 \mathrm{~nm}$. The large compressive stress amounting to $\sim 2.5 \mathrm{GPa}$, present in the $\mathrm{PZO}$ layer near the $\mathrm{PZO}$ and SRO interface could be responsible for the stabilization of a 8-nm-thick uniform layer of rhombohedral PZO in the heterostructures under investigation. However, the stress value reported for a 5-nm thin film is rather speculative. It is also worth mentioning that the Raman spectroscopy measurement yields an average response from the outer $\sim 12$-nm-thick region of the sample and therefore could not resolve the local stress fields that might also be present near the interface between PZO and SRO in the thick PZO films $\left(\mathrm{d}_{\mathrm{PZO}} \geqslant 80 \mathrm{~nm}\right)$.

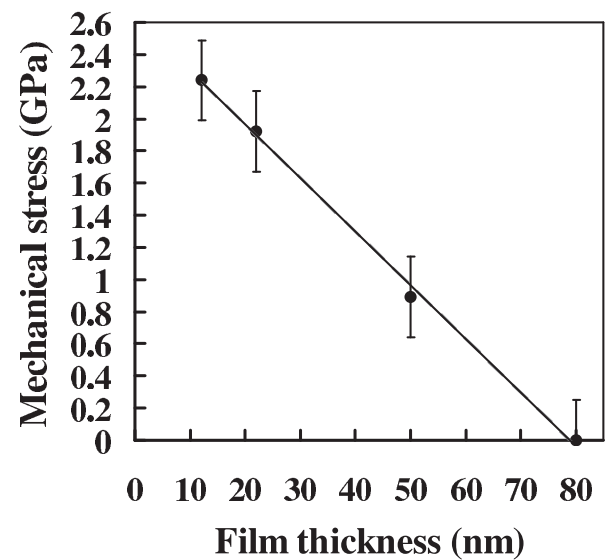

FIG. 6. Plot of the mechanical stress values vs $\mathrm{PbZrO}_{3}$ film thickness. Within the investigated range, the film thickness-stress value correlation is roughly linear. The error bars account for the inaccuracies in the determination of the Raman peak shifts and for the errors induced by the extraction of the calibration data from literature. The solid line is a guide to the eye.
To explain the stabilization of the rhombohedral phase in the thin PZO films investigated in this study, let us consider the temperature-dependent phase transition in PZO. The manifestation of the rhombohedral phase in PZO has been reported earlier in single crystals and high-purity ceramics of the material within a narrow range of temperature near $\mathrm{T}_{\mathrm{C}} \cdot{ }^{20-23}$ In case of thin films, $\mathrm{PZO}$ is likely to grow in the cubic phase at the deposition temperature $\left(\mathrm{T}_{\mathrm{d}} \sim 575^{\circ} \mathrm{C}\right)$. Although the transition temperatures might be quite different in epitaxial PZO thin films, we consider for simplicity the bulk phase transition for $\mathrm{PZO}\left(\mathrm{T}_{\mathrm{C}} \sim 230^{\circ} \mathrm{C}\right)$. When the PZO films are cooled down from the deposition temperature through $\mathrm{T}_{\mathrm{C}}$, PZO may undergo an initial transition from cubic to rhombohedral, followed by a transition to the RT orthorhombic phase. ${ }^{10,20,43}$ Fesenko et al. ${ }^{44}$ established the electric field-temperature phase diagram of PZO and reported the stabilization of rhombohedral PZO over a wide range of electric field and temperature. Topolov et al. ${ }^{45}$ discussed that internal mechanical stress can play a crucial role in stabilizing the rhombohedral phase of PZO due to the small free-energy difference between the orthorhombic and the rhombohedral phases of this material. ${ }^{14-16}$ An energy gain of $\sim 0.235 \mathrm{eV}$ was also estimated for the cubic-rhombohedral phase transition in PZO. ${ }^{32}$ For the thin PZO films $\left(\mathrm{d}_{\mathrm{PZO}} \sim 5-10 \mathrm{~nm}\right.$ ) under investigation, the mechanical stress field present at the PZO and SRO interface could possibly overcome the small freeenergy difference between these two phases of PZO and hence stabilize the rhombohedral phase when passing through $\mathrm{T}_{\mathrm{C}}$, preventing any further transition to the orthorhombic phase. Furuta et $\mathrm{al}$. also reported such a phase transition in $\mathrm{PZO}$ under high values of mechanical stress (similar to the present case). But unfortunately, their report did not include any structural information on the phases. ${ }^{35}$ As the stress field got reduced by increasing $\mathrm{d}_{\mathrm{PZO}}$ in the present case, the bulklike orthorhombic phase of PZO appeared and became abundant in the thicker films $\left(\mathrm{d}_{\mathrm{PZO}} \geqslant 80 \mathrm{~nm}\right)$.

Such a structural evolution of PZO thin films should, in principle, have a significant impact on the electrical properties of the material. To establish the structure-electrical property correlation in these heterostructures, their dynamic P-E responses were studied at RT. Figure 7(a) displays the dynamic P-E hysteresis loops obtained from PZO thin films of
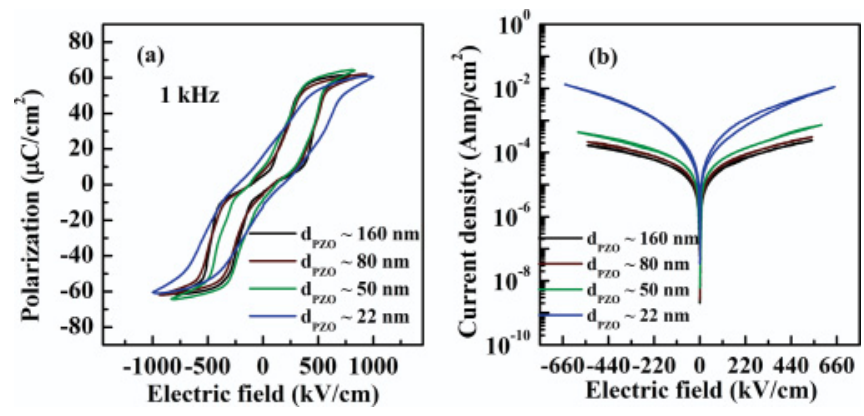

FIG. 7. (Color online) (a) RT P-E hysteresis response of four representative thin films. A clear transition from AFE double loop to FE single loop accompanied by an increase in $\mathrm{P}_{\mathrm{r}}$ was observed by reducing the PZO layer thickness. (b) The leakage current density of the PZO thin films also increased by reducing the PZO layer thickness. 


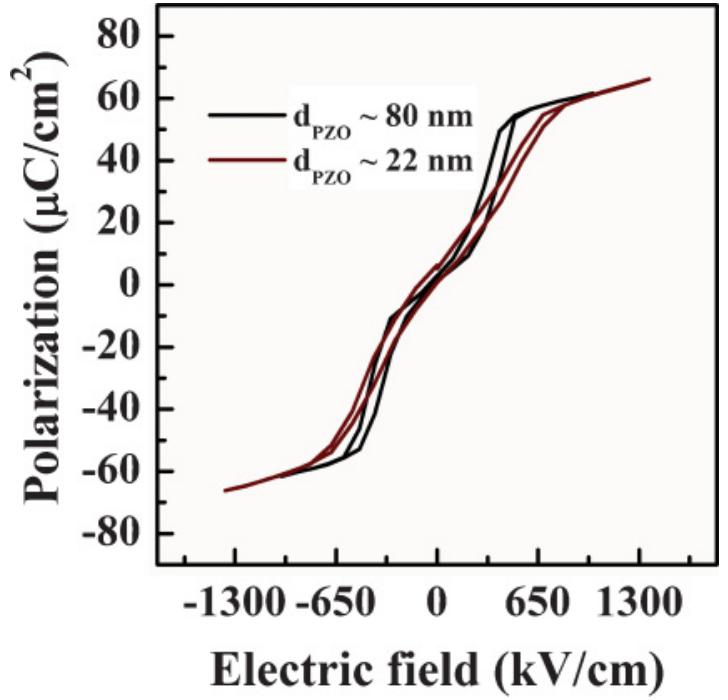

FIG. 8. (Color online) The RT static hysteresis loops measured with a relaxation time of $0.1 \mathrm{~s}$ exhibited a transition from AFE to FE response by reducing the PZO layer thickness.

thickness ranging between 22 and $160 \mathrm{~nm}$ measured at a signal frequency of $1 \mathrm{kHz}$. The double hysteresis loops exhibited by the samples with $\mathrm{d}_{\mathrm{PZO}} \sim 160,80$, and $50 \mathrm{~nm}$ established their AFE nature. Interestingly, all these samples exhibited nonzero in-field $\mathrm{P}_{\mathrm{r}}$ at zero bias, indicating a possible mixed FE-AFE behavior. The in-field $\mathrm{P}_{\mathrm{r}}$ increased monotonically by reducing the PZO layer thickness, and at $\mathrm{d}_{\mathrm{PZO}} \sim 22 \mathrm{~nm}$ a FE-like single hysteresis loop with only spurious track of a double loop was observed. The single hysteresis loop accompanied by a large in-field $\mathrm{P}_{\mathrm{r}}\left(\sim 16 \mu \mathrm{C} / \mathrm{cm}^{2}\right)$ indicated a possible predominant $\mathrm{FE}$ nature of the sample. It is important to note that reduction of $\mathrm{d}_{\text {PZO }}$ also resulted in an increase in the dc leakage current conduction in these heterostructures [Fig. 7(b)]. The large magnitude of leakage current rendered it difficult to measure the P-E responses of the films with $\mathrm{d}_{\mathrm{PZO}}<20 \mathrm{~nm}$.

Polarization vs electric field characteristics of these heterostructures were further investigated by measuring their static hysteresis response. Figure 8 displays the RT static hysteresis response of two representative heterostructures possessing $\mathrm{d}_{\mathrm{PZO}} \sim 80$ and $22 \mathrm{~nm}$. The sample with $\mathrm{d}_{\mathrm{PZO}} \sim 80 \mathrm{~nm}$ showed a double loop, while the sample with $\mathrm{d}_{\mathrm{PZO}} \sim 22 \mathrm{~nm}$ showed a single loop, confirming their respective predominant AFE and FE characteristics. These results are consistent with those observed in the measurements performed in the dynamic mode. The static hysteresis measurements yielded some difference in the values of the $\mathrm{P}_{\mathrm{r}}$ of the samples compared to those measured in the dynamic mode. This could be attributed to the effective relaxation of polarization during the set relaxation time of $0.1 \mathrm{~s}$.

In order to determine the thickness-dependent true remnant polarization characteristics of these PZO thin films, remnant hysteresis measurements were performed on all the heterostructures. Measuring a P-E response before and after performing the remnant hysteresis measurements did not show any significant change in the polarization values confirming that the samples were not fatigued by the voltage cycles. An increase in the $\mathrm{P}_{\mathrm{r}}$ from $\sim 0.4 \mu \mathrm{C} / \mathrm{cm}^{2}$ to $\sim 2.4 \mu \mathrm{C} / \mathrm{cm}^{2}$ (Fig. 9)

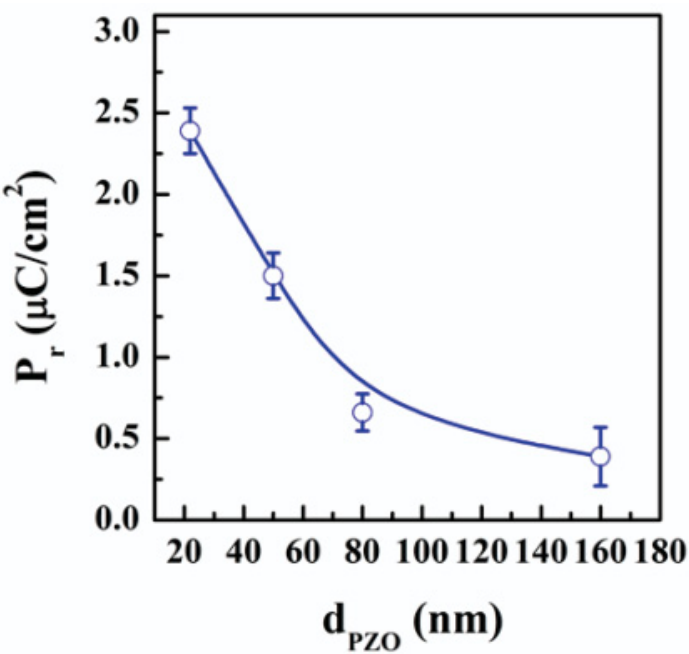

FIG. 9. (Color online) Remnant polarization values obtained from RT remnant hysteresis measurements of four representative $\mathrm{PbZrO}_{3}$ thin films. An increase in the remnant polarization by reducing the PZO layer thickness indicated predominant FE characteristics of the thin PZO films. The solid line is a guide to the eye.

by reducing $d_{\mathrm{PZO}}$ from 160 to $22 \mathrm{~nm}$ further confirmed the enhanced $\mathrm{FE}$ response due to the presence of a larger volume fraction of the $\mathrm{PZO}_{\mathrm{R}}$ phase.

Local ferroelectric properties of these heterostructures were also investigated by PFM measurements. Two samples were selected for this purpose, one showing predominant AFE behavior $\left(\mathrm{d}_{\mathrm{PZO}} \sim 80 \mathrm{~nm}\right)$ and another showing FE response $\left(\mathrm{d}_{\mathrm{PZO}} \sim 22 \mathrm{~nm}\right)$. The in-field hysteresis of the sample with $\mathrm{d}_{\mathrm{PZO}} \sim 80 \mathrm{~nm}$ [Fig. 10(a)] showed a double loop characteristic of AFE materials, while the remnant piezohysteresis displayed no loop [Fig. 10(b)]. In-field measurements of the sample with $\mathrm{d}_{\mathrm{PZO}} \sim 22 \mathrm{~nm}$ [Fig. 10(c)] clearly showed a FE loop,

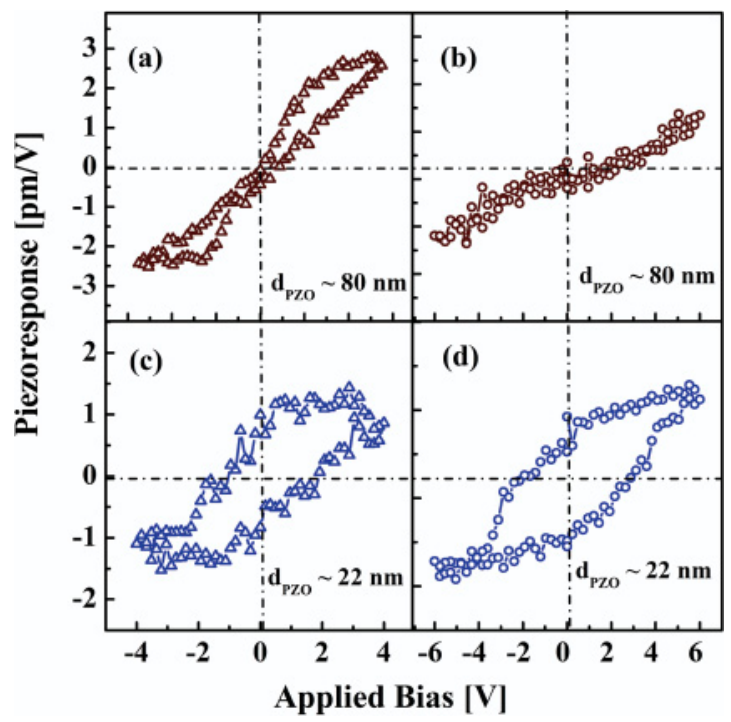

FIG. 10. (Color online) In-field [(a) and (c)] and remnant [(b and (d)] piezoresponse hysteresis loops obtained from two representative heterostructures. The PFM measurements confirm the thicknessdependent predominant $\mathrm{AFE}\left(\mathrm{d}_{\mathrm{PZO}} \sim 80 \mathrm{~nm}\right)$ and $\mathrm{FE}\left(\mathrm{d}_{\mathrm{PZO}} \sim 22 \mathrm{~nm}\right)$ characteristics of the heterostructures. 
and remnant measurements [Fig. 10(d)] further confirmed its predominant FE characteristics. It is important to note that the low signal values obtained in this case are above the noise level of our instrument $(<0.5 \mathrm{pm} / \mathrm{V})$ and hence can be attributed solely to the materials under investigation. These results are in agreement to the macroscopic measurements confirming the thickness dependent RT ferroelectricity in PZO thin films.

\section{SUMMARY}

Epitaxial $\mathrm{PbZrO}_{3}$ thin films of different thickness were fabricated by pulsed laser deposition. With the reduction of $d_{\text {PZO from }} 160$ to $5 \mathrm{~nm}$, the crystal structure of the PZO layer gradually changed from a mixture of orthorhombic and rhombohedral to a pure rhombohedral structure, accompanied by a change in the epitaxial relation from $\mathrm{PZO}(120)_{o} / / \mathrm{SRO} / \mathrm{STO}(100)$ to $\mathrm{PZO}(100)_{R} / / \mathrm{SRO} / \mathrm{STO}(100)$. Detailed microstructural characterization by HRTEM, STEM, and NBED analyses revealed that the rhombohedral phase of PZO was stabilized in the heterostructures in order to accommodate the epitaxial strain imposed by the SRO and STO substrate. RT UV Raman spectroscopic investigations showed that the structural phase transition by reducing the
PZO layer thickness was influenced by a large magnitude of the compressive stress field $(\sim 1.92-2.5 \mathrm{GPa})$. RT polarization hysteresis measurements performed on thicker PZO films $\left(\mathrm{d}_{\mathrm{PZO}} \sim 50-160 \mathrm{~nm}\right)$ resulted in double P-E loops accompanied by an increase in $\mathrm{P}_{\mathrm{r}}$ with reducing the thickness of the PZO layer. A large $\mathrm{P}_{\mathrm{r}}\left(\sim 16 \mu \mathrm{C} / \mathrm{cm}^{2}\right)$ and appearance of a ferroelectric-like single P-E loop for a film with $\mathrm{d}_{\mathrm{PZO}} \sim 22 \mathrm{~nm}$ suggested its predominant $\mathrm{FE}$ behavior. Static hysteresis, remnant hysteresis, and PFM measurements also supported the thickness-dependent transition from predominantly antiferroelectric to predominantly ferroelectric characteristics of the $\mathrm{PbZrO}_{3}$ thin films. The structure-property correlations in the present study established the epitaxial strain-driven ferroelectric phase formation in $\mathrm{PbZrO}_{3}$ thin films.

\section{ACKNOWLEDGMENTS}

The authors acknowledge Prof. Dietrich Hesse for the valuable scientific discussions and for carefully reading the manuscript. A. Roy Chaudhuri acknowledges Alexander von Humboldt Foundation for financial support. *chaudhur@mpi-halle.mpg.de

${ }^{1}$ B. Jaffe, W. J. Cook, and H. Jaffe, Piezoelectric Ceramics (Academic Press, London, 1971), p. 174.

${ }^{2}$ K. Yamakawa, K. Wa. Gachigi, S. Troiler-McKinstry, and J. P. Dougherty, J. Mater. Sci. 32, 174 (1997).

${ }^{3}$ B. Xu, N. G. Pai, and L. E. Cross, Mater. Lett. 34, 157 (1998).

${ }^{4}$ B. Xu, Y. Ye, and L. E. Cross, J. Appl. Phys. 87, 2507 (2000).

${ }^{5}$ X. Li, J. Zhai, and H. Chen, J. Appl. Phys. 97, 024102 (2005).

${ }^{6}$ K. Singh, Ferroelectrics 94, 433 (1989).

${ }^{7}$ A. S. Mischenko, Q. Zhang, J. F. Scott, R. W. Whatmore, and N. D. Mathur, Science 311, 1270 (2006).

${ }^{8}$ J. Parui and S. B. Krupanidhi, Phys. Stat. Sol. (RRL) 2, 230 (2008).

${ }^{9}$ M. E. Lines and A. M. Glass, Principles and Applications of Ferroelectric and Related Materials (Clarendon, Oxford, 1977), p. 262.

${ }^{10}$ E. Sawaguchi, G. Shirane, and Y. Takagi, J. Phys. Soc. Jpn. 6, 333 (1951).

${ }^{11}$ G. Shirane, E. Sawaguchi, and Y. Takagi, Phys. Rev. 84, 476 (1951).

${ }^{12}$ E. Sawaguchi, Ferroelectrics 266, 341 (2002).

${ }^{13}$ H. Fujishita, Y. Shiozaki, and E. Sawaguchi, J. Phys. Soc. Jpn. 46, 1391 (1979).

${ }^{14}$ E. Sawaguchi, J. Phys. Soc. Jpn. 8, 615 (1953).

${ }^{15}$ E. Sawaguchi and T. Kittaka, J. Phys. Soc. Jpn. 7, 336 (1952).

${ }^{16}$ A. P. DeBretteville, Jr., Phys. Rev. 94, 1125 (1954).

${ }^{17}$ F. Jona, G. Shirane, F. Mazzi, and R. Pepinsky, Phys. Rev. 105, 849 (1957).

${ }^{18}$ X. Dai, J. F. Li, and D. Viehland, Phys. Rev. B. 51, 2651 (1995).

${ }^{19}$ L. Pintilie, K. Boldyreva, M. Alexe, and D. Hesse, J. Appl. Phys. 103, 024101 (2008).

${ }^{20}$ B. A. Scott and G. Burns, J. Am. Ceram. Soc. 55, 331 (1972).

${ }^{21}$ V. J. Tennery, J. Electrochem. Soc. 112, 1117 (1965).
${ }^{22}$ V. J. Tennery, J. Am. Ceram. Soc. 49, 483 (1966).

${ }^{23}$ R. W. Whatmore and A. M. Glazer, J. Phys. C 12, 1505 (1979).

${ }^{24}$ P. Ayyub, S. Chattopadhyay, R. Pinto, and M. S. Multani, Phys. Rev. B 57, R5559 (1998).

${ }^{25}$ Z. G. Ban and S. P. Alpay, MRS Symp. Proc. 718, D 10.28 (2002).

${ }^{26}$ I. MacLaren and Z. L. Wang, Appl. Phys. Lett. 80, 1406 (2002).

${ }^{27}$ E. A. Eliseev and M. D. Glinchuk, Physica B. 400, 106 (2007).

${ }^{28}$ K. Boldyreva, L. Pintilie, A. Lotnyk, I. B. Misirlioglu, M. Alexe, and D. Hesse, Appl. Phys. Lett. 91, 122915 (2007).

${ }^{29}$ G. Koster, B. L. Kropman, G. J. H. M. Rijnders, and D. H. A. Blank, Appl. Phys. Lett. 73, 2920 (1998).

${ }^{30}$ D. B. Williams and C. B. Carter, Transmission Electron Microscopy, 2nd ed. (Plenum, New York, 1996), p. 176.

${ }^{31}$ FEI-TRUECRYSTAL STRAIN SOFTWARE ${ }^{\mathrm{TM}}$ (component for the FEI-TEM Imaging \& Analysis Offline software, FEI Company, USA, 2009).

${ }^{32}$ D. J. Singh, Phys. Rev. B 52, 12559 (1995).

${ }^{33}$ H. Lee, Y. S. Kang, S-J. Cho, B. Xiao, H. Morkoc, T. D. Kang, G. S. Lee, J. Li, S-H Wei, P. G. Snyder, and J. T. Evans, J. Appl. Phys. 98, 094108 (2005).

${ }^{34}$ A. E. Pasto and R. A. Condrate, J. Am. Ceram. Soc. 56, 436 (1973).

${ }^{35}$ H. Furuta, S. Endo, L. C. Ming, and H. Fujishita, J. Phys. Chem. Solids 60, 65 (1999).

${ }^{36}$ K. Prume, T. Schmitz, and S. Tiedke, in Polar Oxides, edited by R. Waser, U. Böttger, and S. Tiedke (Wiley-VCH, Weinheim, 2005), p. 63.

${ }^{37}$ M. Dawber, K. M. Rabe, and J. F. Scott, Rev. Mod. Phys. 77, 1083 (2005).

${ }^{38}$ [http://www.ferrodevices.com/1/297/application_notes.asp].

${ }^{39}$ C. Harnagea, A. Pignolet, M. Alexe, D. Hesse, and U. Goesele, Appl. Phys. A. 70, 261 (2000). 
${ }^{40}$ J. W. Matthews and A. E. Blakeslee, J. Cryst. Growth 27, 118 (1974).

${ }^{41}$ K. Yamasaki, Y. Soejima, and K. F. Fischer, Acta Crystallogr. Sec. B 54, 524 (1998).

${ }^{42}$ T. Ohnishi, K. Shibuya, T. Yamamoto, and M. Lippmaa, J. Appl. Phys. 103, 103703 (2008).
${ }^{43}$ D. F. Weirauch and V. J. Tennery, J. Am. Ceram. Soc. 53, 229 (1970).

${ }^{44}$ O. E. Fesenko, R. V. Kolesova, and Y. G. Sindeev, Sov. Phys. Solid State 21, 668 (1979).

${ }^{45}$ V. Yu. Topolov, A. V. Turik, O. E. Fesenko, and V. V. Eremkin, Ferroelectrics Lett. 20, 19 (1995). 